\title{
Diagnostic Potential of Distortion Product Otoacoustic Emissions in Noninvasive Assessment of Elevated Intracranial Pressure: Different Patterns of DPOAE Alterations in the Guinea Pig
}

\author{
Ulrich Strassen, ${ }^{1,2}$ Christoph Deppe, ${ }^{2,3}$ Klaus Mees, ${ }^{3}$ Nikolaus Plesnila, ${ }^{2,4}$ Martin Canis, ${ }^{3}$ \\ Sebastian Strieth, ${ }^{3}$ and Bernhard Olzowy ${ }^{2,3}$ \\ ${ }^{1}$ Department of Otorhinolaryngology, Head and Neck Surgery, Technical University of Munich, Ismaningerstraße 22, \\ 81675 Munich, Germany \\ ${ }^{2}$ Walter Brendel Centre for Experimental Medicine, Ludwig Maximilians University of Munich, Marchioninistraße 15, \\ 81377 Munich, Germany \\ ${ }^{3}$ Department of Otorhinolaryngology, Head and Neck Surgery, Ludwig Maximilians University of Munich, Marchioninistraße 15, \\ 81377 Munich, Germany \\ ${ }^{4}$ Department of Neurosurgery, Ludwig Maximilians University of Munich, Marchioninistraße 15, 81377 Munich, Germany
}

Correspondence should be addressed to Bernhard Olzowy, bernhard.olzowy@med.uni-muenchen.de

Received 31 October 2011; Accepted 5 December 2011

Academic Editors: S. Baris and E. Shipton

Copyright (C) 2011 Ulrich Strassen et al. This is an open access article distributed under the Creative Commons Attribution License, which permits unrestricted use, distribution, and reproduction in any medium, provided the original work is properly cited.

\begin{abstract}
Distortion product otoacoustic emissions (DPOAEs) have been proposed for monitoring the intracranial pressure (ICP) noninvasively. Aim of this study was to establish an animal model in the guinea pig for a detailed characterisation of ICP-related DPOAE alterations. In guinea pigs, the ICP was elevated experimentally and the DPOAE levels were continuously monitored. Two different patterns of DPOAE level changes were observed: (1) a decrease of few decibels affecting mainly the frequency $f_{2}$ $=2 \mathrm{kHz}$ with instant recovery after normalization of ICP, probably related to alterations of middle ear sound transmission; (2) a more pronounced level decrease affecting all frequencies, combined with a second decrease and prolonged recovery after ICP normalization, which might be related to alterations of the cochlear blood flow. Alterations of the levels of DPOAEs might not only provide information about ICP but also indicate critical ICP-induced reductions of the cochlear and the cerebral perfusion pressure.
\end{abstract}

\section{Introduction}

Elevated intracranial pressure (ICP) can complicate the course of several common pathologies such as head injury, intracranial hemorrhage, hydrocephalus, stroke, hypoxic brain injury, central nervous system infection, and acute liver failure. Early diagnosis and treatment of ICP elevation is considered crucial to prevent permanent brain damage, and monitoring the ICP is usually recommended for patients at risk. In the clinical routine, the external ventricular drain and parenchymal probes are commonly used for monitoring the ICP. Both procedures are cost-intensive, invasive, and carry a risk of hemorrhage and infection [1].
One possibility to monitor the ICP noninvasively is an assessment of the perilymphatic pressure by audiologic techniques. The cerebrospinal fluid (CSF) and the perilymph communicate through the cochlear aqueduct, the vestibular aqueduct, and perineural and perivascular spaces [2]. An increase of the CSF pressure, therefore, results in increased pressure on the stapes footplate and, consequently, in an altered sound transmission through the middle ear [3-5], which can sensitively be detected by DPOAEs. In humans, elevation of the ICP induces a decrease of the DPOAE levels most pronounced at the frequencies between 0.75 and $2 \mathrm{kHz}$ while higher frequencies only show minor changes $[3,6,7]$. After ICP elevation, DPOAE level alterations reach their 
plateau in $8-30 \mathrm{~s}$ in humans [3] and $16-18 \mathrm{~s}$ in gerbils [8] with even shorter recovery time.

The cerebral perfusion pressure is the difference between the mean arterial pressure and the ICP. When the cerebral perfusion pressure is reduced below the lower threshold of autoregulation, the cerebral blood flow (CBF) decreases and cerebral ischemia ensues [1]. The same mechanism applies to the cochlear blood flow (CoBF) in relation to the intracochlear pressure [9], and elevation of the ICP instantly leads to elevation of the intracochlear pressure $[10,11]$. Therefore, ICP elevation will reduce both the $\mathrm{CBF}$ and the CoBF when it exceeds their respective lower threshold of autoregulation. DPOAEs have been shown to react very sensitively to transient reduction of the CoBF across all measured frequencies and to exhibit a very characteristic course of level alterations after reperfusion $[12,13]$. Strikingly similar alterations of DPOAE levels were observed due to transient systemic hypoxia [14-16].

It is hypothesized that a continuous increase of the ICP should consecutively produce two distinct patterns of DPOAE changes: firstly, a decrease of the lower frequencies due to altered middle ear sound transmission and-at higher ICP values-a decrease across all measured frequencies due to a critical drop in the CoBF. An animal model was developed to characterize different patterns of DPOAE level changes due to increased ICP. This might help to better interpret DPOAE level alterations observed during ICP monitoring and possibly to derive additional information about critical ICP values leading to a reduction of the CoBF and the CBF.

\section{Materials and Methods}

2.1. Animals, Monitoring of Vital Parameters, and Surgical Preparation. 16 albino guinea pigs weighing $350-520 \mathrm{~g}$ were anesthetized by intramuscular administration of midazolam $(1.0 \mathrm{mg} / \mathrm{kg})$, medetomidine $(0.2 \mathrm{mg} / \mathrm{kg})$, and fentanyl $(0.025 \mathrm{mg} / \mathrm{kg})$. In order to maintain anesthesia, half the dose had to be injected approximately every $45 \mathrm{~min}$ when the heart rate and/or the blood pressure started to rise. The rectal temperature was kept stable at $38 \pm 0.1^{\circ} \mathrm{C}$ using a feedback regulated heating blanket. The animals were arterially catheterized for monitoring the blood pressure. The mean arterial blood pressure remained stable at $49.68 \pm$ $4.39 \mathrm{mmHg}(66.23 \pm 5.85 \mathrm{hPa})$ during the course of the measurements. The capillary oxygen saturation $\left(\mathrm{SaO}_{2}\right)$ was continuously monitored via pulse oximetry. If $\mathrm{SaO}_{2}$ fell below $80 \%$ during the course of a step of ICP elevation, the experiment was terminated.

A craniotomy extending from the sagittal suture medially, the origin of the temporal muscle laterally, the lambdoid suture posteriorly, and the coronal suture anteriorly was drilled, and the dura mater was loosened from the skull around the craniotomy. A cylinder was fixated to the rim of the craniotomy with cyanoacrylate glue for a waterproof seal. The tip of a digital ICP probe (Codman ICP-Express) was inserted into the parenchyma via a burr hole on the contralateral side and fixated with dental cement (Aqualox). The outer ear canal was cleaned, and a small myringotomy was performed under microscopic control in order to prevent a pressure gradient between the outer ear canal and the middle ear. Finally, a silicone tube connected to the DPOAE probe was inserted into the outer ear canal and fixated with cyanoacrylate glue.

The "Principles of laboratory animal care" (NIH publication no. 86-23, revised 1985) were followed. The experimental protocol and use of the animals reported on in this study were approved by the Animal Care Committee of the University of Munich (Reg-Nr. 55.2-1-54-2531-14/05).

\subsection{DPOAE Recordings and ICP Manipulation. DPOAEs} were recorded in a sound attenuated chamber (Industrial Acoustics Company) using an ER-10C probe (Etymotic Research), the DP2000 software (Mimosa Acoustics), and a standard laptop. DPOAE levels were monitored continuously at the frequencies $f_{2}=2,4,8,12$, and $16 \mathrm{kHz}$. At lower frequencies, DPOAEs were not recordable sufficiently above the noise floor. DPOAEs were evoked with one pair of primaries at $f_{2} / f_{1}=1.2, L_{1}=69 \mathrm{~dB} \mathrm{SPL}$, and $L_{2}=60 \mathrm{~dB}$ SPL to produce maximal DPOAE levels [17]. The microphone response was time averaged $(6 \mathrm{~s})$, so that one single measurement point was taken approximately every $6 \mathrm{~s}$ (each frequency every $30 \mathrm{~s}$ ). The levels of the cubic difference tones at $2 f_{1}-f_{2}$ were stored and processed offline.

DPOAE levels were recorded continuously during the course of the whole experiment starting 5 min before elevating the ICP to verify a stable baseline level. Then, the cylinder was filled stepwise with $0.9 \%$ sodium chloride solution in order to elevate the ICP to defined pressure values $(18,22$, and $24 \mathrm{mmHg}=24,29$, and $32 \mathrm{hPa}$ ). These pressure values were chosen, because preliminary experiments had revealed, that no measurable DPOAE level changes occurred below $18 \mathrm{mmHg}$ and that the animals did not tolerate ICP values above $24 \mathrm{mmHg}$ well. The cylinder was emptied after each pressure step for 10 min to verify stability of DPOAE levels at normal pressure. After recalibration of the probe, DPOAE levels were again recorded for $5 \mathrm{~min}$ before elevating the ICP again.

Six animals had to be excluded from further data processing because ICP could not be kept stable due to fluid leakage between the cylinder and the craniotomy. In ten animals a total of 25 ICP intervals were recorded, where the desired pressure value could be kept stable in the range of $\pm 2 \mathrm{mmHg}$ for $4-7 \mathrm{~min}$. In two animals, the experiment was terminated at $22 \mathrm{mmHg}$, and in one animal, at $24 \mathrm{mmHg}$ due to a drop in oxygen saturation, which explains the total number of 25 ICP intervals instead of 30 (Table 1).

2.3. Data Processing. Only DPOAE measurements with a signal-to-noise ratio of $>6 \mathrm{~dB}$ were used for the further data processing. DPOAE measurements during unstable ICP while filling or emptying the cylinder, defined by a variation $>2 \mathrm{mmHg}$ from the baseline or the target value, respectively, were discarded.

For every animal and each frequency, the arithmetic mean (mean) and the standard deviation (SD) of the DPOAE levels were calculated for each period with elevated ICP (mean ${ }_{\mathrm{ICP}}$, and $\left.\mathrm{SD}_{\mathrm{ICP}}\right)$ and for each period with normal 
TABLE 1: Overview of all measured ICP intervals with stable ICP in the range of $\pm 2 \mathrm{mmHg}$ of the target value indicated in the table and the information whether they were valid. For valid ICP intervals the pattern of DPOAE level alterations is given. For explanation of the two patterns see Figure 1. Note that the second pattern only occurred at the respective highest ICP value.

\begin{tabular}{|c|c|c|c|}
\hline Animal no. & $\mathrm{ICP}[\mathrm{mmHg}]$ & Valid & Pattern \\
\hline \multirow{3}{*}{1} & 18 & yes & first \\
\hline & 22 & yes & first \\
\hline & 24 & yes & second \\
\hline \multirow{3}{*}{2} & 18 & $\mathrm{no}^{1}$ & \\
\hline & 22 & yes & first \\
\hline & 24 & yes & first \\
\hline \multirow{3}{*}{3} & 18 & yes & no change \\
\hline & 22 & yes & first \\
\hline & 24 & yes & second \\
\hline \multirow{2}{*}{4} & 18 & yes & \multirow{2}{*}{ first } \\
\hline & 22 & no $^{2}$ & \\
\hline \multirow{3}{*}{5} & 18 & yes & first \\
\hline & 22 & yes & first \\
\hline & 24 & yes & second \\
\hline \multirow{2}{*}{6} & 18 & yes & \multirow[b]{2}{*}{ no change } \\
\hline & 22 & $\mathrm{no}^{2}$ & \\
\hline \multirow{3}{*}{7} & 18 & no ${ }^{1}$ & \multirow{3}{*}{ first } \\
\hline & 22 & no ${ }^{1}$ & \\
\hline & 24 & yes & \\
\hline \multirow{3}{*}{8} & 18 & $\mathrm{no}^{1}$ & \multirow{3}{*}{ no change } \\
\hline & 22 & yes & \\
\hline & 24 & no $^{1}$ & \\
\hline \multirow{3}{*}{9} & 18 & yes & \multirow{3}{*}{$\begin{array}{c}\text { first } \\
\text { second }\end{array}$} \\
\hline & 22 & yes & \\
\hline & 24 & $\mathrm{no}^{2}$ & \\
\hline \multirow{3}{*}{10} & 18 & yes & first \\
\hline & 22 & yes & first \\
\hline & 24 & yes & second \\
\hline
\end{tabular}

${ }^{1}$ invalid because mean $n_{\text {postICP }}$ did not stabilize in the range of $\pm 2 \mathrm{SD}_{\text {baseline }}$

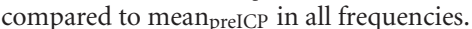

${ }_{2}^{2}$ invalid because $\mathrm{SaO}_{2}$ dropped below $80 \%$. In these cases, no further pressure steps were recorded.

See Section 2.3 for calculations and inclusion criteria.

ICP before and after an ICP interval (mean preICP, $\mathrm{SD}_{\text {preICP, }}$ mean $_{\text {postICP }}$ and $\mathrm{SD}_{\text {postICP }}$ ). Additionally, the $\mathrm{SD}$ for all measurements with normal ICP was calculated as measure for the baseline stability of the DPOAE levels of the specific animal at a given frequency $\left(\mathrm{SD}_{\text {baseline }}\right)$.

An alteration of the DPOAE levels during ICP elevation in the single animal for a specific frequency was regarded significant, when $\mid$ mean $_{I C P}-\operatorname{mean}_{\text {preICP }} \mid>2 \mathrm{SD}_{\text {baseline. Data }}$ from an interval with elevated ICP and significant alterations of the DPOAE level was only used for further processing if the arithmetic mean stabilized in the range of two times the $\mathrm{SD}_{\text {baseline }}$ (i.e., $\mid$ mean $_{\text {postICP }}-\operatorname{mean}_{\text {preICP }} \mid<2 \mathrm{SD}_{\text {baseline }}$ ) after lowering the ICP. However, during experiments with transient clamping of the labyrinthine artery, characteristic DPOAE level alterations occur after reperfusion $[12,13]$, and identification of similar level alterations after pressure normalization was the aim of this study. Therefore, mean $_{\text {postICP }}$ was calculated from measurements after potential postischemic level alterations had abated. This strict stability criterion was necessary to unequivocally attribute the observed small level alterations to variations of the ICP, because other factors may have caused alterations of the DPOAE levels in our complex experimental setting with long anesthesia. Five ICP intervals did not fulfill this stability criterion in all measured frequencies and were discarded (Table 1). During all of the remaining 20 ICP intervals, single frequencies did not fulfill the stability criterion. This may have been due to small variations of the DPOAE probe in the outer ear canal affecting only single frequencies. In these cases, these frequencies were discarded, but the other valid frequencies were used for the final data analysis. This explains why the number of valid ICP intervals per frequency in Tables 2(a) and 2(b) do not sum up to 20 (Tables 2(a) and 2(b)).

Data from 20 intervals with elevated ICP in ten animals were included into the main data analysis. The DPOAE levels and the ICP were plotted versus time for each interval and each measured frequency.

The alterations of the DPOAE levels described due to elevated ICP affect only the lower frequencies, occur 8-30 s after elevation of the ICP, and recover even more quickly after lowering the ICP, with stable DPOAE levels when the ICP is stable $[3,8]$. In contrast, the alterations of the DPOAE levels due to hypoxia affect all frequencies and are characterized by a pronounced instability $[14,15]$ during hypoxia and characteristic level changes for five to ten minutes after reoxygenation [14-16]. The data was visually screened for the second pattern and divided into two groups which underwent a separate descriptive analysis.

A Wilcoxon signed rank test was performed $(\alpha=0.05)$ to compare mean DPOAE levels before and during ICP elevation of all ICP intervals for the different frequencies that did not exhibit the second pattern. Only five ICP intervals exhibited the second pattern so that these data were not amenable to statistical analysis.

Microsoft Excel was used for figure creation.

\section{Results}

The final data analysis encompassed ten animals and 20 intervals with elevated ICP. Two clearly distinct patterns of DPOAE level alterations were observed.

In 12 intervals, the maximum of the observed changes was reached immediately after the ICP had reached a stable value and then remained stable until the ICP was lowered. Stable DPOAE levels in the range of the levels before ICP elevation could be measured immediately after normalization of the ICP (Figure 1(a)). This pattern will further be referred to as the first pattern. There were a total of three ICP intervals that did not exhibit significant level alterations in any of the measured frequencies. These data were grouped and statistically analyzed together with the first pattern. Although there were single intervals with significant 


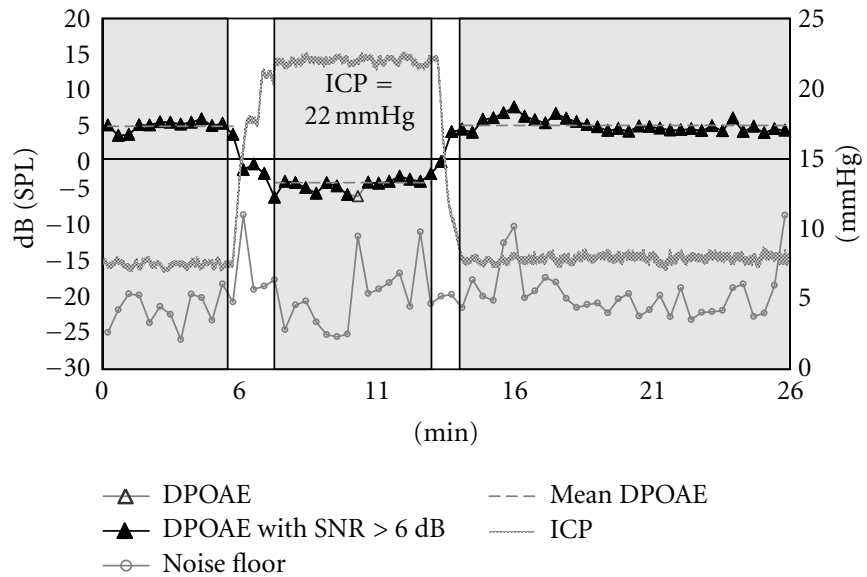

(a)

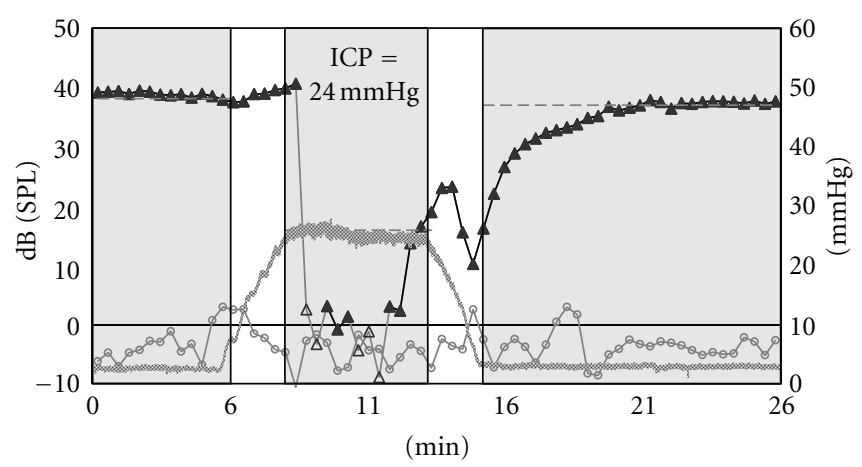

$\triangle$ DPOAE
$\leftarrow$ DPOAE with SNR $>6 \mathrm{~dB} \quad$ Mean DPOAE
- ICP

(b)

FIGURE 1: DPOAE levels (left vertical axis) and ICP (right vertical axis) plotted versus time for one pressure step in a single animal. The shaded areas mark data sets with stable ICP included into the final data analysis. The horizontal dotted lines mark the mean DPOAE level before ICP elevation $\left(\right.$ mean $\left._{\text {preICP }}\right)$, under elevated ICP $\left(\right.$ mean $\left._{I C P}\right)$, and after ICP normalization $\left(\operatorname{mean}_{\text {postICP }}\right)$. For the second pattern, mean $_{\text {postICP }}$ was calculated after the supposedly postischemic alterations of the DPOAE levels had abated. (a) First pattern of DPOAE level changes, attributed to altered middle ear sound transmission. Note the prompt level decrease with ICP elevation, the stable DPOAE levels during constant ICP, and the instant recovery of the DPOAE levels after ICP normalization $\left(f_{2}=2 \mathrm{kHz}\right)$. (b) Second pattern of DPOAE level changes, that might be attributed to reduced CoBF. Note the more pronounced level decay and the instability thereafter. After ICP normalization, the DPOAE levels start to recover but then decrease again sharply before they slowly recover to pre-ICP values $\left(f_{2}=12 \mathrm{kHz}\right)$.

changes of the DPOAE levels at higher frequencies, the mean change of the DPOAE levels for all these intervals with elevated ICP was only significant at $f_{2}=2 \mathrm{kHz}(P=0.011)$. The mean change of the DPOAE levels was $-3.17 \mathrm{~dB}$ with a maximal change of $-8.05 \mathrm{~dB}$. SD ICP was generally small and never exceeded $2.5 \mathrm{~dB}$ (Table $2(\mathrm{a})$ ).

Five animals exhibited a different pattern of DPOAE alterations at the respective highest ICP value, consisting of a much larger decay and instability of the DPOAE levels during elevated ICP affecting all frequencies. After normalization of the ICP, the DPOAE levels started to recover but then decreased again sharply. After reaching a minimum, the DPOAE levels recovered steadily to pre-ICP values over a time of approximately $5 \mathrm{~min}$ (Figure 1(b)). This pattern will further be referred to as the second pattern. It was never observed at ICP $<22 \mathrm{mmHg}$ (Table 1 ). $\mathrm{SD}_{\text {ICP }}$ was much larger and always exceeded $2.5 \mathrm{~dB}$ (Table 2(b)).

\section{Discussion}

4.1. Pathophysiologic Interpretation of the Two DPOAE Alteration Patterns. Our study in guinea pigs showed two clearly distinct patterns of DPOAE level alterations due to elevated ICP. One consisted in a level decay of few decibels mainly affecting the frequency $f_{2}=2 \mathrm{kHz}$. DPOAE levels decreased and recovered without a detectable delay after elevating and lowering the ICP, respectively (Figure 1(a)). This first pattern has been described by many authors and is commonly explained by pressure transduction to the inner ear fluid compartment with an increased hydrostatic load on the stapes footplate leading to increased stiffness of the stapes system and consequently to an altered sound transmission through the middle ear [3].
The second pattern showed a much larger decay and instability of the DPOAE levels affecting all frequencies and a prolonged course of DPOAE level alterations after lowering the ICP, where the DPOAE levels started to recover but then decreased again sharply. After reaching a minimum, DPOAE levels recovered steadily to pre-ICP values over a time of approximately $5 \mathrm{~min}$ (Figure 1(b)). A similar phenomenon with equal time course has been described due to reoxygenation after transient hypoxia in guinea pigs [1416] and due to reperfusion after transient clamping of the labyrinthine artery in gerbils [12] and rabbits [13]. The complex hypoxic and posthypoxic alterations are not fully understood but might be best explained by a drop of the endocochlear potential following oxygen depletion and consecutive osmotic phenomena $[14,16]$. In our animal model, the increase of the ICP might have led to a decrease of the CoBF. The cerebral perfusion pressure is the difference between the mean arterial pressure and the ICP. When the cerebral perfusion pressure is reduced below the lower threshold of autoregulation $(40-50 \mathrm{mmHg}$ in humans), the CBF decreases [1]. The same mechanism applies to the CoBF-which is also autoregulated in relation to the intracochlear pressure [9]. Changes of the ICP result in changes of the intracochlear pressure in few seconds in guinea pigs $[10,11]$. Suzuki et al. performed direct measurements of ICP, intracochlear pressure, endocochlear potential, CoBF, and CBF during experimental increase of the ICP in guinea pigs. At an ICP of $22 \mathrm{mmHg}$, endocochlear potential, CoBF, and CBF remained stable but decreased at an ICP of $29 \mathrm{mmHg}$. The decrease in $\mathrm{CBF}$ was generally more pronounced than in CoBF [10]. In our study, the second pattern of DPOAE alterations was never observed at an ICP $<22 \mathrm{mmHg}$. In conclusion, the second pattern of DPOAE alterations closely 
TABLE 2: Mean and range of ICP-induced DPOAE level changes $(\triangle \mathrm{DP})$, range of the standard deviation during elevated ICP $\left(\mathrm{SD}_{\mathrm{ICP}}\right)$, and number of valid intervals for different frequencies $f_{2} \cdot$ Mean $_{\triangle \mathrm{DP}}$, range $\mathrm{D}_{\Delta \mathrm{DP}}$, and range $\mathrm{SD}_{\mathrm{ICP}}$ are given in dB SPL. See Section 2.3 for calculations and inclusion criteria. Significant changes are indicated by an asterisk.

(a) First pattern of DPOAE level alterations attributed to altered sound transmission. Note that there is a significant DPOAE level decrease only at $f_{2}=$ $2 \mathrm{kHz}$

\begin{tabular}{lccccc}
\hline & $f_{2}=2 \mathrm{kHz}$ & $f_{2}=4 \mathrm{kHz}$ & $f_{2}=8 \mathrm{kHz}$ & $f_{2}=12 \mathrm{kHz}$ & $f_{2}=16 \mathrm{kHz}$ \\
\hline Mean $_{\mathrm{DDP}}$ & $-3.17^{*}$ & -0.69 & -0.13 & +0.20 & -0.53 \\
Range $_{\Delta \mathrm{DP}}$ & $-8.05-+1.13$ & $-4.42-+1.43$ & $-1.26-+1.28$ & $-0.63-+2.51$ & $-1.55-+0.61$ \\
Range $_{\mathrm{SD}}$ & $0.83-1.66$ & $0.35-2.06$ & $0.34-0.74$ & $0.36-1.33$ & $0.21-1.13$ \\
Number of valid intervals & 10 & 12 & 14 & 14 & 13 \\
\hline
\end{tabular}

(b) Second pattern of DPOAE level alterations that might be attributed to reduced CoBF. Note the pronounced DPOAE level decrease at all measured frequencies and the much larger SDICP

\begin{tabular}{lccccc}
\hline & $f_{2}=2 \mathrm{kHz}$ & $f_{2}=4 \mathrm{kHz}$ & $f_{2}=8 \mathrm{kHz}$ & $f_{2}=12 \mathrm{kHz}$ & $f_{2}=16 \mathrm{kHz}$ \\
\hline Mean $_{\triangle \mathrm{DP}}$ & -2.80 & -7.66 & -9.76 & -11.47 & -12.36 \\
Range $_{\triangle \mathrm{DP}}$ & $-1.53--3.98$ & $-1.97--13.46$ & $-3.67--14.25$ & $-5.25--22.18$ & $-4.44--21.36$ \\
Range $_{\mathrm{SD}}$ & $3.83-7.36$ & $3.74-12.84$ & $3.00-10.24$ & $7.27-17.51$ & $5.25-13.56$ \\
Number of valid intervals & 4 & 5 & 5 & 5 & 5 \\
\hline
\end{tabular}

resembles the DPOAE alterations observed due to hypoxia or reduced CoBF and occur at ICP values that have been shown to affect the CoBF in guinea pigs. It might, therefore, be interpreted as a sign of reduced CoBF.

\subsection{Potential Implications for a Noninvasive ICP Monitoring} with DPOAEs. The individual pattern of the DPOAE levels at different frequencies is highly variable among different ears but remains fairly stable in repeated measurements of the same ear [18]. Therefore, only relative information about changes of the ICP can be read from DPOAE alterations. Even this is only possible in situations, where a baseline measurement at normal ICP can be performed. Measurements during the further course can be either performed punctually or as continuous monitoring. Furthermore, the frequencies at which DPOAEs shall be monitored have to be determined. Our study showed that elevation of the ICP can produce two different patterns of DPOAE alterations. A monitoring protocol should be designed to recognize the different patterns.

Previous studies about DPOAEs under the influence of ICP elevation focused on the first pattern of DPOAE alterations that mainly affects the lower frequency range $[3,6-8,19]$. The most recent study even measured only DPOAEs at $f_{2}=1 \mathrm{kHz}$ [19]. In contrast, we would favor a protocol that at least measures one higher frequency, for example, in humans $4 \mathrm{kHz}$, which should not be affected by the conductive changes due to ICP but should change along with a decrease of the CoBF.

Furthermore, our study identified the stability of the DPOAE levels during repeated measurements as a measure to distinguish between changes due to alteration of middle ear sound transmission and due to a decrease of the CoBF. The SD of the DPOAE levels during elevated ICP $\left(\mathrm{SD}_{\mathrm{ICP}}\right)$ was always $<2.5 \mathrm{~dB}$ at all frequencies in the first (Table $2(\mathrm{a})$ ) but $>2.5 \mathrm{~dB}$ at all frequencies in the second pattern of DPOAE alterations (Table 2(b)). It has to be considered that the stability of DPOAE levels is not an independent measure but depends on the signal to noise ratio (SNR), with decreasing stability when the SNR decreases. The DPOAE level decrease in the second pattern was larger compared to the first pattern, so that a decreased SNR might have contributed to the observed destabilization of the DPOAE level. Systemic hypoxia produced DPOAE level alterations strikingly similar to the second pattern in our study - which suggests common physiologic processes - and the destabilization was observed independent of a decrease of the DPOAE level $[14,15]$. Furthermore, the degree of destabilization observed seems unlikely to result from reduced SNR alone. Therefore, we would favor a continuous monitoring of the DPOAE levels to record the baseline stability of the DPOAE levels at the monitored frequency in order to recognize a destabilization of the DPOAE levels possibly indicating reduced CoBF.

The effects of elevated ICP on the CoBF and the CBF seem to be interrelated with the CBF being even more vulnerable in a guinea pig model [10]. Therefore, signs of a reduced CoBF during monitoring with DPOAEs might also indicate a critical reduction of the CBF.

For a future clinical noninvasive monitoring of the ICP with audiologic measures, the DPOAE levels have some disadvantages. The observed alterations are small (in the range of few $\mathrm{dB}$ ) and have to be detected in a noisy environment with possible confounding factors, such as altered middle ear pressure and ototoxic drugs. The alterations of the DPOAE phases due to elevated ICP might provide valuable additional information $[3,7,8,19]$. Unfortunately, the software used for our study does not provide phase information. A general disadvantage of DPOAEs is that they are only measurable when the inner ear function is normal. The phases of cochlear microphonics have been shown to provide information about the ICP similar to the DPOAE phases but to be measurable also in patients with high-frequency sensorineural hearing loss [19]. The combined information of DPOAE levels, 
DPOAE phases, and cochlear microphonic phases might better allow for a noninvasive ICP monitoring in the future. Studies how DPOAE phases and cochlear microphonic phases change due to a reduction of the CoBF are warranted.

\section{Disclosure}

This work was presented at an Oral Presentation at the 8th Congress of the German ENT Society 2009 (preliminary data).

\section{Conflict of Interests}

The authors declare that they have no conflict of interests.

\section{References}

[1] L. C. Padayachy, A. A. Figaji, and M. R. Bullock, "Intracranial pressure monitoring for traumatic brain injury in the modern era," Child's Nervous System, vol. 26, no. 4, pp. 441-452, 2010.

[2] R. R. Ciuman, "Communication routes between intracranial spaces and inner ear: function, pathophysiologic importance and relations with inner ear diseases," American Journal of Otolaryngology, vol. 30, no. 3, pp. 193-202, 2009.

[3] B. Büki, A. Chomicki, M. Dordain et al., "Middle-ear influence on otoacoustic emissions. II: contributions of posture and intracranial pressure," Hearing Research, vol. 140, no. 1-2, pp. 202-211, 2000.

[4] R. Kast, "A new method for noninvasive measurement of short-term cerebrospinal fluid pressure changes in humans," Journal of Neurology, vol. 232, no. 4, pp. 260-261, 1985.

[5] I. Klockhoff, G. Anggård, and L. Anggård, "Recording of cranio-labyrinthine pressure transmission in man by acoustic impedance method," Acta Oto-Laryngologica, vol. 61, no. 4, pp. 361-370, 1966.

[6] A. M. Frank, C. Alexiou, P. Hulin, T. Janssen, W. Arnold, and A. E. Trappe, "Non-invasive measurement of intracranial pressure changes by otoacoustic emissions (OAES) - a report of preliminary data," Zentralblatt fur Neurochirurgie, vol. 61, no. 4, pp. 177-180, 2000.

[7] S. E. Voss, M. F. Adegoke, N. J. Horton, K. N. Sheth, J. Rosand, and C. A. Shera, "Posture systematically alters earcanal reflectance and DPOAE properties," Hearing Research, vol. 263, no. 1-2, pp. 43-51, 2010.

[8] B. Büki, E. De Kleine, H. P. Wit, and P. Avan, "Detection of intracochlear and intracranial pressure changes with otoacoustic emissions: a gerbil model," Hearing Research, vol. 167, no. 1-2, pp. 180-191, 2002.

[9] T. Nakashima, S. Naganawa, M. Sone et al., "Disorders of cochlear blood flow," Brain Research Reviews, vol. 43, no. 1, pp. 17-28, 2003.

[10] T. Suzuki, T. Nakashima, and N. Yanagita, "Effects of increased cerebrospinal fluid pressure on cochlear and cerebral blood flow," European Archives of Oto-Rhino-Laryngology, vol. 250, no. 6, pp. 332-336, 1993.

[11] E. O. Thalen, H. P. Wit, J. M. Segenhout, and F. W. J. Albers, "Dynamics of inner ear pressure change caused by intracranial pressure manipulation in the guinea pig," Acta Oto-Laryngologica, vol. 121, no. 4, pp. 470-476, 2001.

[12] T. Mom, P. Avan, R. Romand, and L. Gilain, "Monitoring of functional changes after transient ischemia in gerbil cochlea," Brain Research, vol. 751, no. 1, pp. 20-30, 1997.
[13] T. Mom, F. F. Telischi, G. K. Martin, and B. L. LonsburyMartin, "Measuring the cochlear blood flow and distortionproduct otoacoustic emissions during reversible cochlear ischemia: a rabbit model," Hearing Research, vol. 133, no. 1-2, pp. 40-52, 1999.

[14] B. Olzowy, G. Von Gleichenstein, M. Canis, N. Plesnila, and K. Mees, "Complex level alterations of the 2f1-f2 distortion product due to hypoxia in the guinea pig," European Archives of Oto-Rhino-Laryngology, vol. 265, no. 11, pp. 1329-1333, 2008.

[15] B. Olzowy, G. Von Gleichenstein, M. Canis et al., "Level alterations of the $2 \mathrm{f} 1-\mathrm{f} 2$ distortion product due to hypoxia in the guinea pig depend on the stimulus frequency," European Archives of Oto-Rhino-Laryngology, vol. 267, no. 3, pp. 351-355, 2010.

[16] G. Rebillard, J. F. L. Klis, M. Lavigne-Rebillard, P. Devaux, J. L. Puel, and R. Pujol, "Changes in 2f1-f2 distortion product otoacoustic emissions following alterations of cochlear metabolism," British Journal of Audiology, vol. 27, no. 2, pp. 117121, 1993.

[17] C. E. Michaelis, D. D. Gehr, K. Deingruber, W. Arnold, and K. Lamm, "Optimum primary tone level setting for measuring high amplitude DPOAEs in guinea pigs," Hearing Research, vol. 189, no. 1-2, pp. 58-62, 2004.

[18] W. Wagner, G. Heppelmann, R. Vonthein, and H. P. Zenner, "Test-retest repeatability of distortion product otoacoustic emissions," Ear and Hearing, vol. 29, no. 3, pp. 378-391, 2008.

[19] B. Büki, F. Giraudet, and P. Avan, "Non-invasive measurements of intralabyrinthine pressure changes by electrocochleography and otoacoustic emissions," Hearing Research, vol. 251, no. 1-2, pp. 51-59, 2009. 


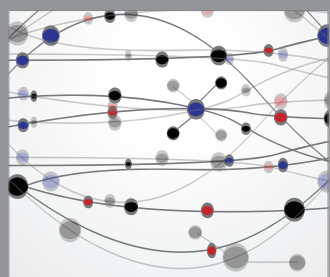

The Scientific World Journal
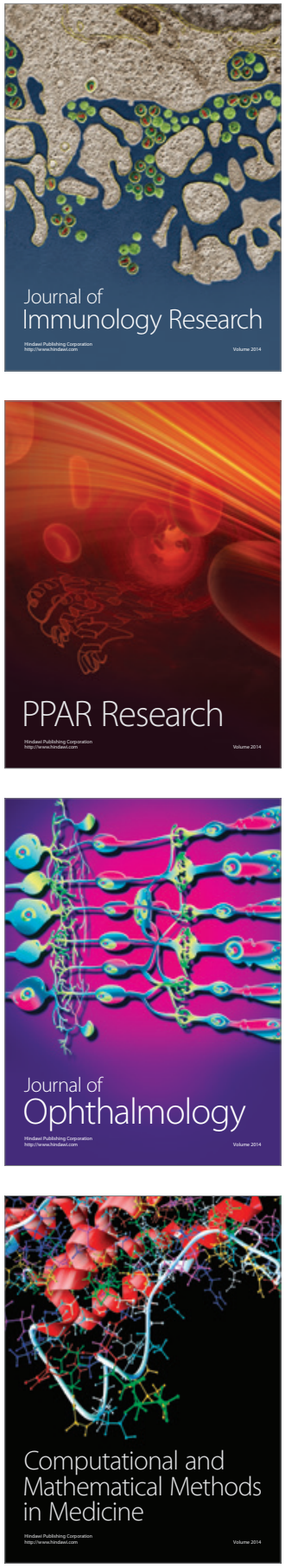

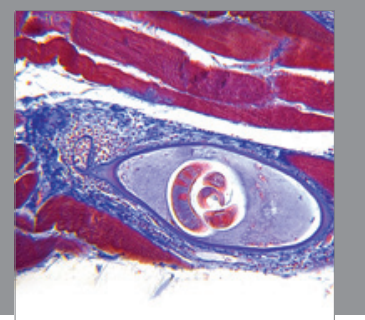

Gastroenterology

Research and Practice
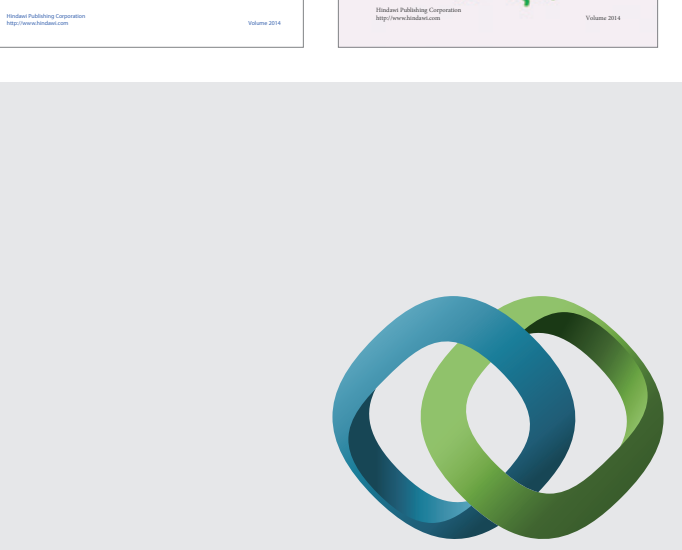

\section{Hindawi}

Submit your manuscripts at

http://www.hindawi.com
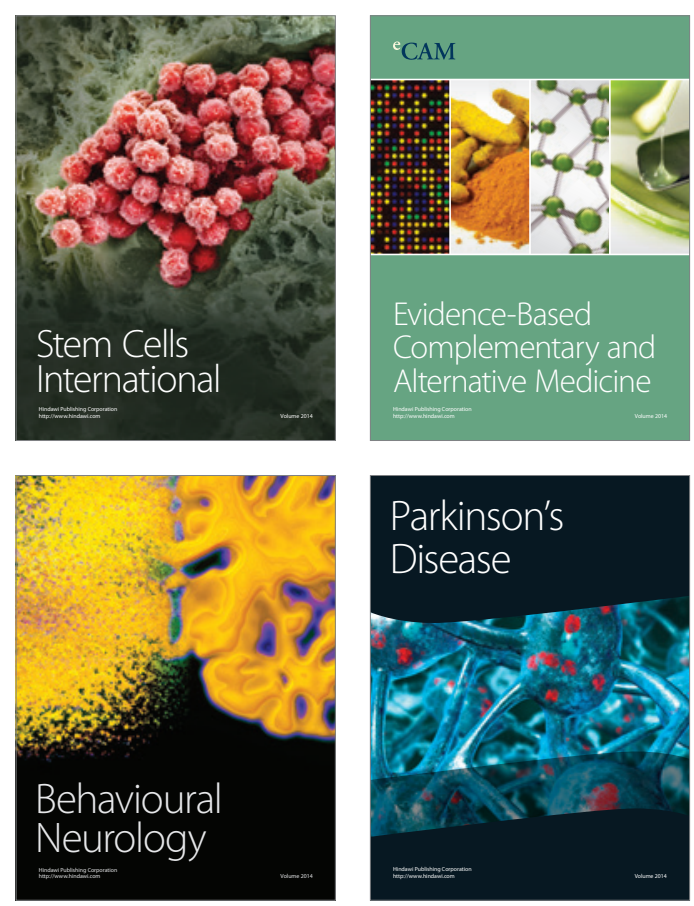

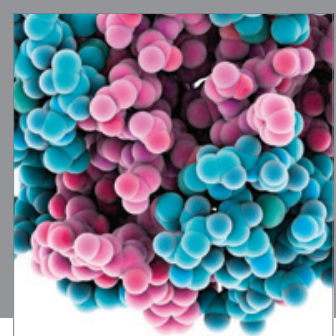

Journal of
Diabetes Research

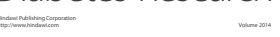

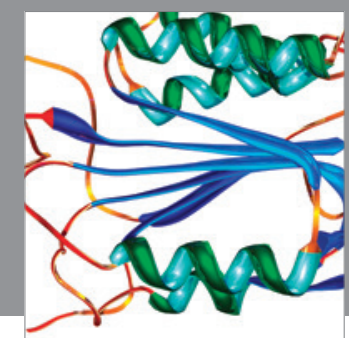

Disease Markers
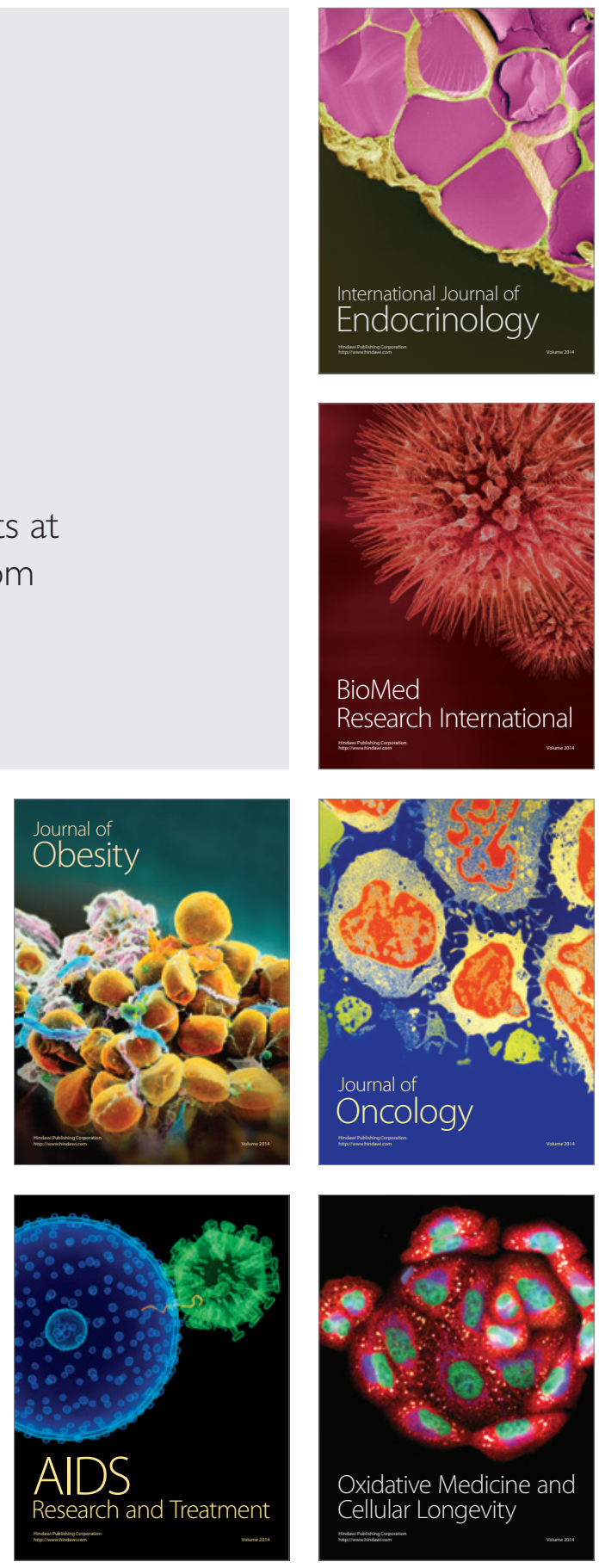